\title{
Spatial Hypertext for End-User Development Tools
}

\author{
Daniel Roßner* \\ Hof University \\ Institute of Information Systems \\ Hof, Germany \\ daniel.rossner@iisys.de
}

\author{
Claus Atzenbeck \\ Hof University \\ Institute of Information Systems \\ Hof, Germany \\ claus.atzenbeck@iisys.de
}

\begin{abstract}
Software applications become more and more dominant in our daily life and work. However, it is very difficult to provide sophisticated tools for all arising use cases. End-User Development (EUD) is a term that describes the development of applications by end users rather than professional developers. This enables them creating highly specialized solutions.

End users are laypersons when it comes to building software, therefore they need appropriate tools for managing the whole development process. This includes designing, implementing and deploying applications. While there are already various tools available, we focus on a recommendation feature for graphical EUD tools, utilizing their spatial hypertext capabilities.

In this paper we provide an overview of some common issues such tools are often struggling with. We explain how visually analyzing the workspace, parsing an implicit spatial hypertext and eventually presenting recommendations may tackle them. We further describe the project HEIMDALL in detail, especially the way of generating recommendations for software modules with the aim to raise users' awareness. Furthermore, we discuss the use of such a system that reaches a similar understanding of relationships between software modules as users have. Finally, we point to open issues that still need to be addressed to improve results and their presentation.
\end{abstract}

\section{CCS CONCEPTS}

- Human-centered computing $\rightarrow$ Hypertext / hypermedia; Graphical user interfaces; • Software and its engineering $\rightarrow$ Software creation and management;

\section{KEYWORDS}

open hypermedia systems; CB-OHS; spatial hypertext; Mother; End-User Development; HCI

ACM Reference Format:

Daniel Roßner and Claus Atzenbeck. 2018. Spatial Hypertext for End-User Development Tools. In HUMAN '18: 1st Workshop on Human Factors in Hypertext, July 9, 2018, Baltimore, MD, USA. ACM, New York, NY, USA, 7 pages.

https://doi.org/10.1145/3215611.3215612

\footnotetext{
${ }^{*}$ Corresponding author

Permission to make digital or hard copies of part or all of this work for personal or classroom use is granted without fee provided that copies are not made or distributed for profit or commercial advantage and that copies bear this notice and the full citation on the first page. Copyrights for third-party components of this work must be honored.

For all other uses, contact the owner/author(s).

HUMAN '18, fuly 9, 2018, Baltimore, MD, USA

( 2018 Copyright held by the owner/author(s).

ACM ISBN 978-1-4503-5658-9/18/07.

https://doi.org/10.1145/3215611.3215612
}

\section{INTRODUCTION}

More and more tasks in our daily life are - and will be - managed by software. However, whereas the usability of that software improved drastically over the past years, the process of its development remains the same. The great complexity of modern software development demands skilled and professional developers. While this might not change in the future, we experience a growing need of laypersons to extend existing applications or to create their own [18]. The term End-User Development (EUD) refers to the techniques, tools and methods used to accomplish this [11]. An end user in this context is a person, who "is programming to achieve the result of a program primarily for personal, rather public use" [10]. Hence, the main difference between professional and end-user development refers to the type of achievement, rather than the actual skill of a developer.

There are various motivations for end users to get into EUD. It may be the need of specialized solutions for domain specific problems, fast changing requirements [16] or the lack of easy-to-use professional solutions. Powerful EUD tools enable users to handle the described scenarios. Quite a few of the existing tools are heavily based on graphical representations of the program's logic, because they want to "reduce the cognitive burden of learning by shrinking the conceptual distance between actions in the real world and programming" [8]. Even though many software tools do not support visual programming for various reasons (e. g., spreadsheets), we will focus on graphical ones.

The well known, yet abandoned, service Yahoo! Pipes was a modern example of a graphical EUD tool. It provided modules to retrieve content from the web, manipulate and finally aggregate it. The modules were organized in a global library. Users could place them in their canvas and model a data flow by connecting the modules with pipes. Other examples of visual programming tools are VisPro [22], WebMakeup [7], or ResEval Mash [9].

All those graphical EUD tools have something in common: The user has to:

(1) find basic building blocks, e. g., control structures like loops, complex software modules, or web resources;

(2) arrange them in the $n$-dimensional space provided by the tool; and

(3) describe what the program is supposed to do with the building blocks.

Once the logic is modeled using explicit connections the resulting structure can be interpreted as navigational hypertext, consisting of nodes and links. Most of today's systems are exclusively interested in explicit structures, fully ignoring the spatial arrangement of modules on the space. The user, however, is able to interpret 
the implicit structure and, thus, takes advantage of additional relationship information. For example, the user recognizes the relation between items that are arranged as vertical list; the computer does not. This leads to a knowledge gap between user and machine: The user has a different, more complex understanding of the information space than the machine. This reduces the level of collaboration and assistance that would be possible otherwise.

This type of structure is known as spatial hypertext. Relations are expressed by the visual appearance of nodes or by their spatial arrangement [12]. In order to reach a common understanding of the structure, the machine must be able to interpret the relationships between items on the space as a human user would do. This can be provided by highly specialized machines, so-called spatial or visual parsers. For instance, a specific parser may compute relationships considering only the spatial arrangement of items. Another parser may consider visual commonalities among items, such as color, shape, size, or orientation. There even may be parsers that consider the user's editing process in order to detect relationships between items $[19,20]$. A machine that has a similar view of a visually represented knowledge than a human user combines human-like assistance with access to large data resources.

For Bonnie Nardi the value of a human assistant is that he works on a task and anticipates the next steps without the need of explicitly explaining everything in detail. In 1993 she wrote: "The hope is that the computer will be able to do the same" [15]. Our paper shows how a system which is using spatial hypertext to assist the user fosters creativity, productivity and simplicity of EUD in graphical based EUD tools. The HEIMDALL project is used as an example case to present our experiences and work in supporting EUD with capabilities of spatial hypertext combined with machine intelligence to reach a common understanding of the implicit structures represented on a space.

\section{ANALYSIS}

In the past years much research efforts went into the development of sophisticated EUD tools of different kinds. Some of them are specific to a domain, like ResEval Mash, others try to be applicable in a broader view according to the area of application. There are also tools like Scratch ${ }^{1}$ that do not focus on "getting things to work", but rather on the process of programming itself to educate children. In the following analysis we have collected some common issues related to EUD tools that can be solved by interpreting users' spatially arranged information.

[14] and [16] demand design tools that offer a low threshold to get started as novice user without limiting experts to use them professionally. This requirement conflicts with the tool's general applicability to cover a wide range of domains [9]. It is true that not all three requirements can be fulfilled in a completely satisfying manner, however, developers of such tools do not have to give up generality as a whole. Novices could benefit from an advanced supporting feature, that is able to learn from skilled users. Using spatial hypertext may help to implement such a feature, without limiting the domain of the tool.

Growing end user projects (in size and complexity) draw the need to share "source code" with other developers or even cooperate with

\footnotetext{
${ }^{1}$ https://scratch.mit.edu/
}

them to improve or enhance the application. Such a cooperation may be very challenging, considering different qualifications, skills, and interests of the users [13]. Laypersons and professionals should be able to cooperate in the same project without impeding each other. It may be necessary to provide different levels of details depending on the user's skills. For example, a novice user is only working with graphical elements, while a more skilled user can edit the functionality behind those. In general, graphical tools are easier to master, because they are familiar to end users, who only use graphical applications in their daily life. Spatially arranged elements can serve as a means of communication between users with different skill levels.

Especially laypersons tend to skip the process of planning and designing an application before they start implementing it, because planning the implementation implies prior, sophisticated knowledge of the EUD tool. This and fast changing requirements raise the need to modify the software without the need of reprogramming whole parts of it $[11,17]$. Taking the previous argument into account, modifications have to be recognizable for other developers. While even professional projects sometimes lack of a good documentation, this is all the more true for EUD. The creation of implicit structures can help to draft first ideas more easily, because users are not forced to phrase definite behavior. With support of a recommendation system, based on these structures, novices get valuable feedback on their activities. Furthermore, the concept of using modules, which can be assembled in graphical tools, foster the encapsulation of code. Developers of that modules have to think carefully of interfaces and re-usability. As a result, the users are able to organize reusable components within a spatial area, incrementally improving the quality of their project. Since the structure of such a project is presented visually, changes are recognizable at any given time.

Another critical aspect of authoring an application from scratch is the early stage of development. In this phase the user only has a rough idea of what he/she wants to create. Moreover, the user may not know how the EUD tool can help in doing so. Those design tasks are cognitively intense and mostly lead to a trial-and-error approach [21]. Ideally, the tool supports the user in these early stages by giving helpful feedback and allowing him to try out different approaches flexibly. It is important that the user does not get frustrated; instead, it is necessary to keep the user motivated by supporting a continuous progress. As stated above, focusing on implicit, spatial structures enables a supporting tool to help users, before they are able to enunciate their idea. The potentially infinite workspace allows to try out things "somewhere else" and return back immediately - the creation and drafting processes are merging.

An EUD tool that encapsulates logic or functionality into single modules must permit the user to select the desired feature set [13]. This may be provided, for example, by the tool's graphically represented space, by clicking a button, or some metadata (e. g., the package declaration). Most systems offer a text-based search, helping users to find modules they know by name or metadata. Novice users often face the problem that they do not know the modules they are in need of. Beside this, the process of searching using text is a disruptive task, forcing the user to interrupt his creating process. There should be a way to black box missing modules, 


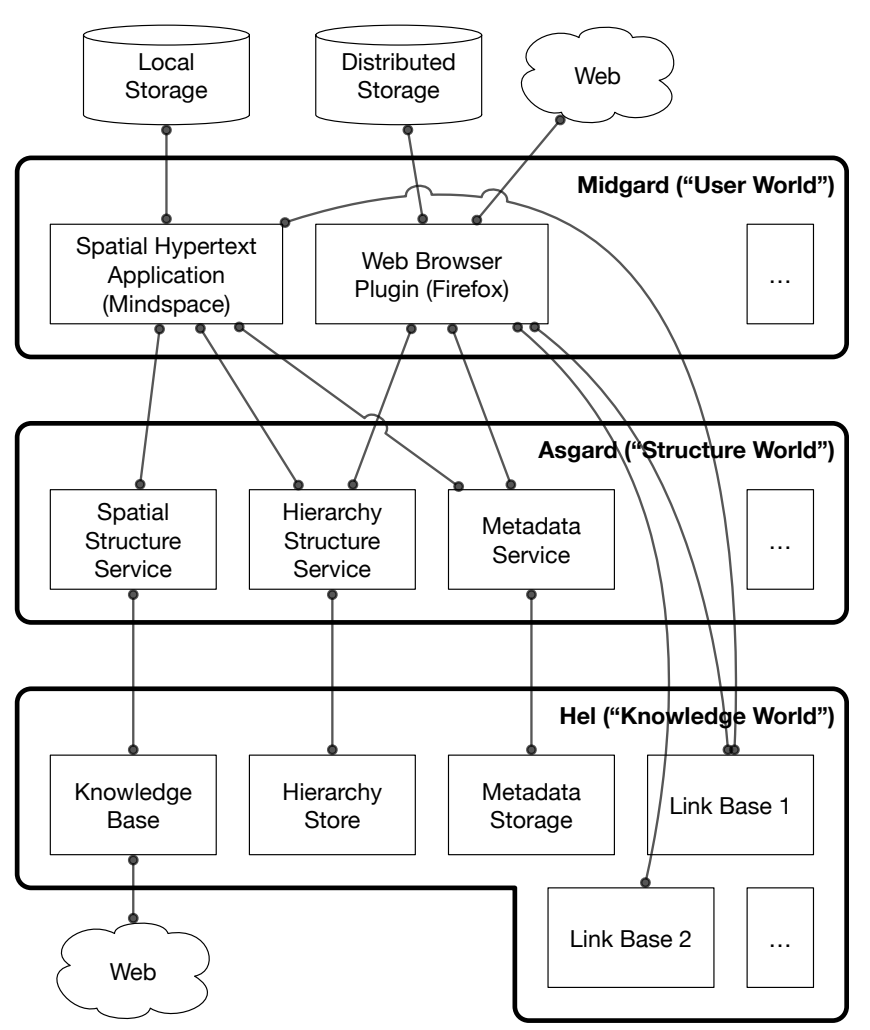

Figure 1: Mother's architecture (exemplary set-up)

such that the user can continue his work and is remembered to identify the missing building blocks at a later point of time.

\section{EXAMPLE CASE: HEIMDALL}

\subsection{Project Description}

The main goal of the ODIN ("Open Data Innovation") project is to create a platform for sophisticated developers as well as for professional users (e. g., journalists) or laypersons who need to work with open data. ODIN's sub-project HEIMDALL focuses on an intelligent, Web-based user interface. The term intelligent refers to the context described in Sect. 2. A typical use-case may include:

(i) the collection of some publicly available weather and traffic accidents data; (ii) the aggregation of this data; and (iii) the generation of a visual representation.

Developers are mainly involved in devising new components, while journalists may want to research specific data to be presented in a newspaper article. Laypersons may want to experiment with data for their personal education or interest. The categorization of end users is not specific or limited to one of the mentioned. Instead, ODIN aims at providing support to people with different prior knowledge or skill levels.

People who want to work with open data often face many different data sources, all with their own API and file format. For IT laypersons it is nearly impossible to handle those sources. For these users, ODIN offers ready-to-use components for accessing, manipulating, or visualizing data. Those are called Cubbles; they are based on Meme Media Technology and previously known as Webbles [cf. 1]. The "Browser-based Development Environment" (BDE) enables the user to arrange Cubbles on a 2D space. Cubbles have slots for input and output, which can be interconnected using pipes. Figure 2 depicts a screenshot of the BDE showing the graphical implementation of a music player using Cubbles. Cubbles are Web components, implemented in JavaScript. They are executed in the user's Web browser, which loads all required modules from the Cubbles Base, a global repository. This makes them highly reusable software components.

The Cubbles technology is not domain specific to open data. Its capability is limited by the available Cubbles. It is possible to create new Cubbles at any time, however, this cannot be done graphically and thus is difficult for laypersons.

\subsection{Implementation}

The BDE is a client implementation for end users to work with the Cubbles platform. When first developed, there was neither the intention to integrate a recommender system nor to become an innovative example case for an EUD tool. Basic features covered the $2 \mathrm{D}$ space, placing, and aggregation of Cubbles, which can be searched by name. While it would have been possible to implement assistance directly or via a dynamic library, we chose to offer a flexible hypertext infrastructure. This broadens the features that can be offered to the client and demonstrates the generality of our component-based open hypermedia system (CB-OHS), called Mother. Figure 1 depicts the overall architecture and some components and their communication as examples. It consists of three basic layers:

(1) Midgard: This is Mother's application layer. It includes components with user interfaces or those that show similar functionalities.

(2) Asgard: This layer holds structure services and related, partly intelligent components.

(3) Hel: Finally, this layer subsumes knowledge-based components, including link bases, knowledge bases, or software that can populate those.

In the past, most CB-OHS proposed navigational hypertext as the most dominant structure type. On the contrary, Mother's architecture "has been developed primarily with one of the most complex (and for machines most difficult to 'understand') hypertext structures in mind. This approach appreciates the specific demands of implicit structures rather than explicit ones." [6] Asgard's spatial structure service analyzes implicit structures using multiple, highly specialized parsers, including those for evaluating spatial, visual, or temporal properties. The result is similar to a human interpretation of the knowledge space, as described in [19, 20]. Further technical details of Mother are described in [6].

In the early stages of this project we observed that users had problems in discovering modules of which they did not know that they were offered by the repository. They had to know the Cubbles' names or search according to their features. Since they did not have knowledge about what they should be looking for, it became difficult for them to accomplish their tasks. The less knowledge a user had about the platform, the harder it was for him/her to discover useful components. 


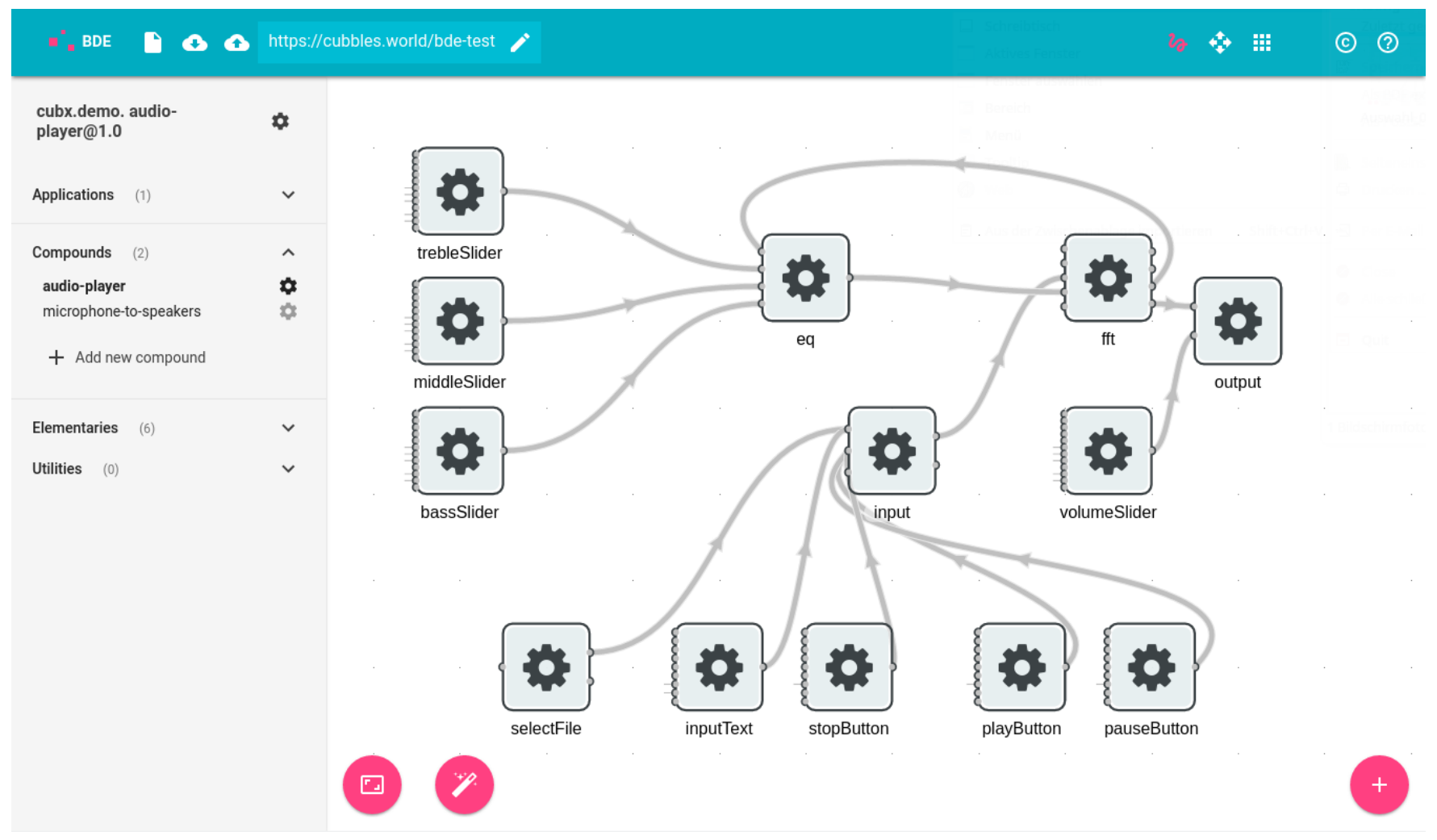

Figure 2: BDE workspace with Cubbles modeling a music player

This problem can be targeted by introducing recommendations. Instead of only providing text-based search, the system should work like a skilled colleague, looking at what the user is doing and proposing relevant Cubbles automatically. For the user it should feel like he is in a pair-programming session with his own computer.

As already mentioned earlier, the user's workspace can be seen as a hypertext, more precisely, as a combination of multiple kinds of hypertextual structures:

(1) Cubbles are also interconnected by their metadata, such as the same versions or the same author.

(2) The linked (i. e., piped) Cubbles relate to navigational hypertext, where data flows (i. e., navigates) to other Cubbles.

(3) Last but not least the visual appearance, like the color, size, or position, refer to spatial hypertext.

For each of the identified structure type, a specialized service was implemented. They keep track of what the user is doing and provide appropriate feedback. The spatial hypertext feature received special attention within the HEIMDALL project, because it perfectly matches the BDE workspace requirements and supports the interpretation of Cubbles structures on it.

At this point we have a system which recognizes all changes to the visual workspace made by the user. Based on that, it generates a complete graph, in which the used Cubbles form the nodes. The result of the analysis, which is provided by our spatial and visual parsers, build weighted edges between the nodes. Those have values between 0 (i. e., no visual/spatial commonalities) and 1 (i. e., very strong visual/spatial relation). Details about the algorithms behind the parsers are provided by [19]. After the parsing process, all edges below a specified threshold are removed from the graph, resulting in one ore more islands of interconnected nodes. Thereafter those are sent to a knowledge base to query related recommendations.

In order to generate useful answers, the knowledge base needs to be fed with some data in advance. In HEIMDALL this happens by looking at preexisting Cubbles in the Cubbles base. Those were developed by experts and used in a reasonably number of projects. Connecting Cubbles result in a higher level Cubble, a so-called compound Cubble, which then can be reused in different contexts.

In order to get an idea of how those Cubbles are used by skilled developers, we analyzed their usage of compound modules. Consider two basic Cubbles; the first one gets an URL as input and outputs an HTML document, the second one takes HTML as input and outputs whether the HTML is valid or not. A developer wants to create an HTML validation service ${ }^{2}$, and thus adds the two mentioned Cubbles to his workspace. After that, he connects the output slot of the first, with the input slot of the second Cubble. To embed his new service into a Web page, he connects the output slot of Cubble wrapping an input field to the HTML loading module. The result of the validation goes to the input slot of a text label. All in all he used four Cubbles to achieve the desired result and pushed the validation service (which is a Cubble itself) to a global repository. The knowledge base scans Cubbles repositories for such

\footnotetext{
${ }^{2}$ See, e. g., https://validator.w3.org/
} 


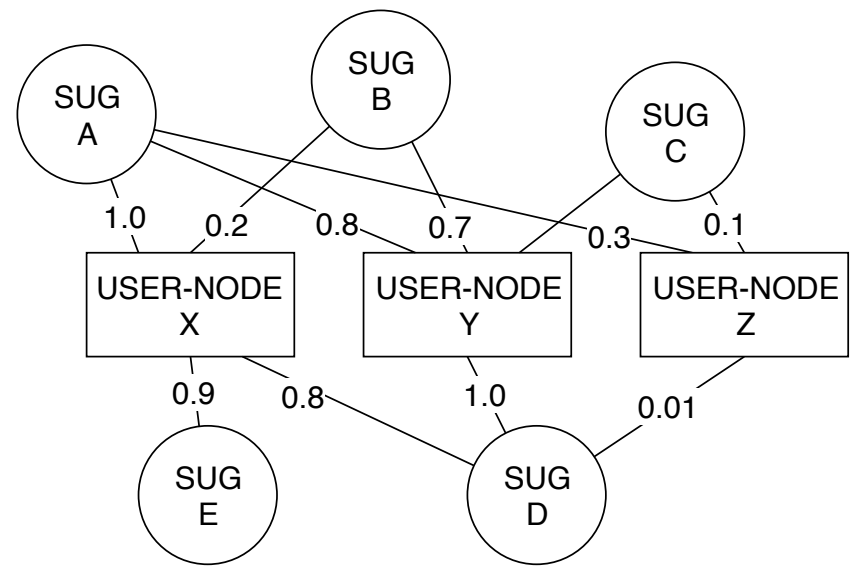

Figure 3: User-nodes X, Y, Z with suggestions (SUG) A, B, C, $D, E$ and accompanying edge values

newly added compounds, looking for explicitly connected Cubbles. Every time two Cubbles are used in combination, the graph-based knowledge base strengthens the edge between them. This is comparable to what the spatial/visual parsers do by analyzing the user's workspace:

(1) The spatial/visual parsers build a graph from implicit visual attributes or spatial locations of Cubbles on the user's workspace.

(2) The knowledge base analyzes released compound Cubbles for interlinked software modules and builds a graph, based on the frequency of their occurrence.

With this information, the knowledge base is able to find related software modules and recommends those to the user.

Because spatial hypertext is basically a set of "visually linked" nodes, the knowledge base has to calculate recommendations for this set rather than single Cubbles. To do so, it looks for nodes with edges to all Cubbles in the set and returns them together with accompanying edge values. While this approach produces helpful results for small sets, it leads to problems with larger sets. That is because possible recommendations have to be linked to the whole set. We tackled this issue with repeating a request several times with a reduced subset. These requests are valued with lower priority to prize the fact, that they do not cover the whole set. The results of the requests are merged and sent to the client as graph, containing the set of requested nodes together with the recommended nodes and related edge values. Figure 3 shows three nodes, placed by the user together with five generated suggestions. If they were no requests on subsets, the system would recommend $\mathrm{A}$ and D only.

Once the client application receives the suggestions, it is responsible for presenting the results to the user. A simple solution would be to take all recommended Cubbles, build the mean value of the edges between them and all user nodes, and put them into a list (sorted by the mean), as shown in Fig. 4. However, this approach does not address the issues described in Sect. 2. While it offers help to the user, it is not clear where the suggestions belong to exactly. This is a problem in particular for large projects, because the user would face a large number of recommended Cubbles. Furthermore, the user would have to interrupt his/her task to visit the list and search for relevant Cubbles within the appropriate context. In the BDE we call this a cluster (even though Cubbles can be visual related without forming a cluster).

A critical issue is the lack of immediate feedback when making changes to the spatial hypertext. A list view is unsuitable to give feedback in real time and at the specific location recommended Cubbles belong to. For this reasons we decided to spatially integrate the suggestions in the workspace. This solves the problem of identifying the context and it is possible to update suggestions simultaneously to the user's actions. At the time of writing, this feature is not yet implemented in the BDE, however, we already are experimenting with different so-called spatial layouters in other clients with similar features (see Sect. 4). The current layout process does not include positioning without overlapping only, but tries to find a semantic meaningful bounding box for each recommendation. While the spatial parser translates implicit structures to a graph with explicit edge values, a spatial layouter does this vice versa: taking an explicit structure and generating an intuitively understandable, visual representation. Please note that placing recommended Cubbles on the space must not alter any user added nodes.

\section{CONCLUSION AND FUTURE WORK}

During the term of the project, we received a lot of feedback from project partners and test users. All of them agreed, that a recommendation system is a big advantage over a text-based search, if it evaluates the users' activities on the workspace. Even skilled developers of the Cubbles platform experience a lack of clarity when it comes to finding required software modules. The implicit generation of suggestions help to explore available modules while interacting with the system. Spatial/visual parsing of the workspace lead to satisfying results - recommendations matched users' expectations, when using a test set of Cubbles. We had to create a test set, because the number of existing Cubbles was way to small to generate a meaningful knowledge base. While this paper is focused on workspace analyzes, the quality of generated results is dependent on the quality of the knowledge base as well. A beneficial base needs a suitable big data source (e. g., many Cubbles and different projects using them) and a domain specific data mining process. For HEIMDALL, there exists a thoughtful implemented, but by far not perfect mining process. For the future we hope to improve this and to support the creation of mining processes in general (e. g., by providing a graphical tool).

As stated in Sect. 3.2, calculated suggestions are presented in a list, located at the left sidebar. This solution breaks the users' workflow, since they have to move their focus away from their actual spatial hypertext. The idea arose to integrate recommendations into the workspace; they basically become an important part of the hypertext. Spatial/visual parsers analyze implicit structure types they are meant for and generate an explicit linked structure, where links do have a weight to take the original implicitness into account. To position suggestions, this process has to be inverted: A linked structure with weighted links, generated by the knowledge base, has to be transformed into a meaningful spatial/visual structure. 


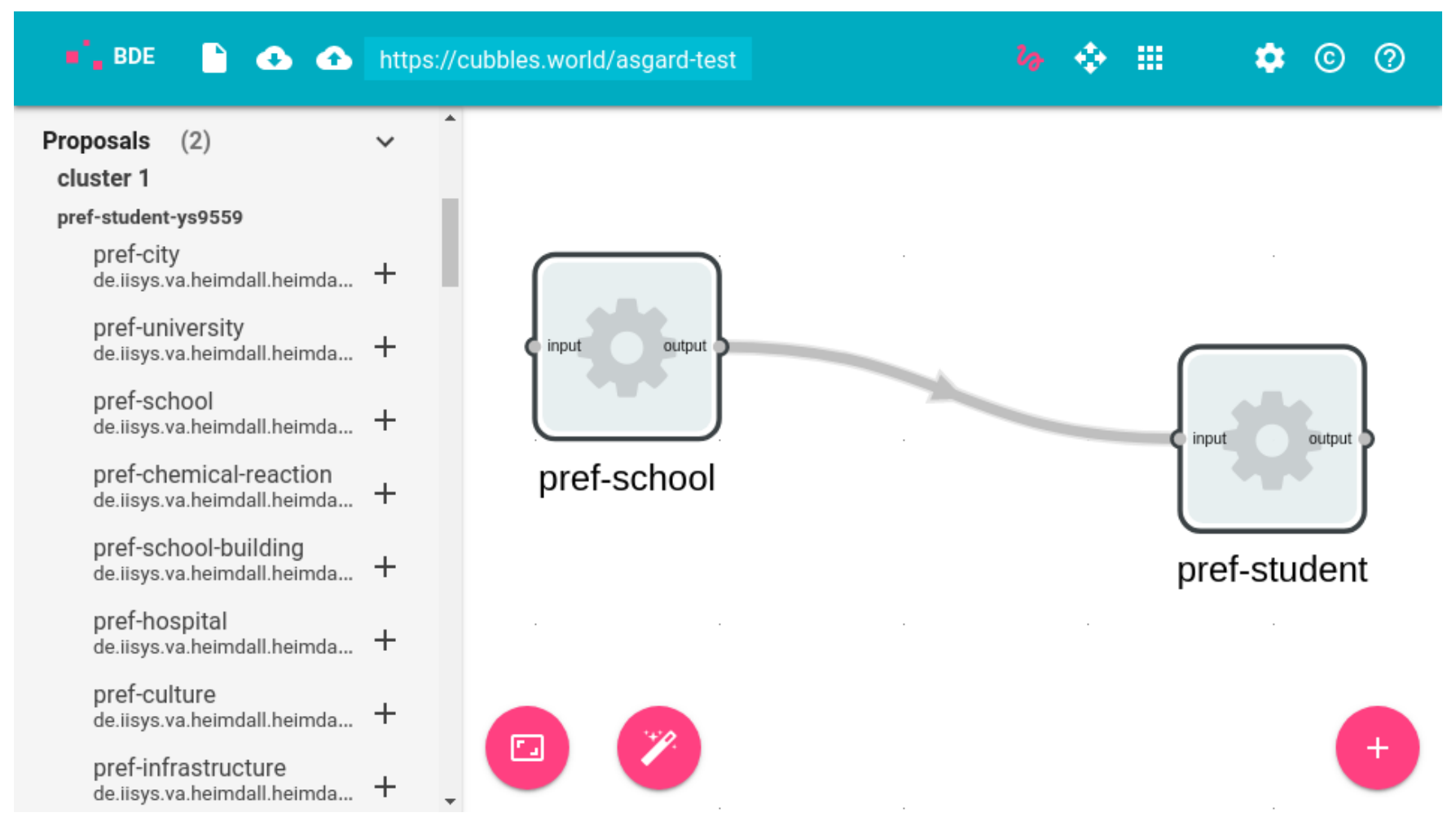

Figure 4: List with recommended Cubbles based on two test Cubbles ("student" and "school”)

Because it is a critical task to interoperate with user generated structure, we are demanding two basic requirements:

(1) User-placed nodes must not change their position in any case.

(2) Suggestions must not change the semantics of users' hypertext.

To get an impression of how a humans would position suggestions in different situations and contexts, we issued short test sheets so some students and colleagues. They were asked to mark the position of given suggestions into a given spatial hypertext with given nodes. While this questionnaire was not broad enough to produce robust results, we saw that all testers tried to use spatial distance as the main factor to model relations. A suggestion, strongly related to a user's node, was positioned close to. With this result in mind, we developed some prototypes, which we call spatial layouter in general. Even though they are functional yet, there are still some fundamental issues to solve in the future. The main problem is to define the context of positioning and to take it into account. One part of the context is defined by the nodes, the user placed explicitly - but that is simply not enough. Suggestions must be positioned without overlapping each other, hence suggestions themselves are part of the context. Furthermore, positioning does not happen on a static workspace. When the user is dragging one of his nodes, spatial and visual properties of the hypertext are changed as well, which may lead to other suggestions and changed positions for the old. Without respecting previous positions as part of the context, small changes to the hypertext may result in completely different appearance of presented suggestions. Addressing these topics will be the next step to build a complete and consistent recommendation solution, which can easily be integrated in many other tools of various domains.

In a long run we aim at providing an EUD environment that is easy to use by laypersons. It should enable them to build sophisticated software tools. Furthermore, our system aims at closing the gap between end user and software developer such that the end user is capable of providing a first prototypic implementation of the desired tool which then may be improved by professionals.

Software development is just one of many application domains that we address with our system Mother. In particular its capability of handling spatial structures makes it a generic problem solving tool in various areas, including police investigations $[2,4]$ and intelligence analysis [3].

Even though we started out with spatial structures [6], our research goes along traditional hypertext research by addressing various hypertext structure domains, including navigational [5] and taxonomic hypertext, argumentation support, and metadata. This makes the HEIMDALL project one building block among others in our overall research.

\section{ACKNOWLEDGMENTS}

This work is part of the ODIN project, sub-project HEIMDALL, funded by the German Federal Ministry of Education and Research (grant ID 03PSWKPD). 


\section{REFERENCES}

[1] Oksana Arnold, Wolfgang Spickermann, Nicolas Spyratos, and Yuzuru Tanaka (Eds.). 2013. Webble Technology. First Webble World Summit (WWS). Communications in Computer and Information Science, Vol. 372. Springer.

[2] Claus Atzenbeck, David L. Hicks, and Nasrullah Memon. 2009. Supporting Emergent Knowledge and Team Communication in Police Investigations. In Proceedings of the Intelligence and Security Informatics Conference (ISI). 95-100. http://dx.doi.org/10.1109/ISI.2009.5137278

[3] Claus Atzenbeck, David L. Hicks, and Nasrullah Memon. 2011. Supporting Reasoning and Communication for Intelligence Officers. International fournal of Networking and Virtual Organisations (IfNVO), Special Issue on Open Source Intelligence and Web Mining (OSINT-WM) 8, 1/2 (2011). http://dx.doi.org/10.1504/ IJNVO.2011.037159

[4] Claus Atzenbeck, Fatih Ozgul, and David L. Hicks. 2009. Linking and Organising Information in Law Enforcement Investigations. In Proceedings of the 13th Inter national Conference on Information Visualization (IV'09). IEEE Computer Society, 443-449. http://dx.doi.org/10.1109/IV.2009.107

[5] Claus Atzenbeck, Daniel Roßner, and Manolis Tzagarakis. 2018. Mother - An Integrated Approach to Hypertext Domains. In Proceedings of the 29th ACM Conference on Hypertext and Social Media. ACM Press. https://doi.org/10.1145/ 3209542.3209570

[6] Claus Atzenbeck, Thomas Schedel, Manolis Tzagarakis, Daniel Roßner, and Lucas Mages. 2017. Revisiting Hypertext Infrastructure. In Proceedings of the 28th ACM Conference on Hypertext and Social Media (HT '17). ACM, New York, NY, USA 35-44. https://doi.org/10.1145/3078714.3078718

[7] Oscar Díaz, Iñigo Aldalur, Cristóbal Arellano, Haritz Medina, and Sergio Firmenich. 2016. Web Mashups with WebMakeup. In Rapid Mashup Development Tools, Florian Daniel and Cesare Pautasso (Eds.). Springer International Publishing, Cham, 82-97.

[8] G. Fischer, E. Giaccardi, Y. Ye, A. G. Sutcliffe, and N. Mehandjiev. 2004. Metadesign: A Manifesto for End-user Development. Commun. ACM 47, 9 (Sept. 2004), 33-37. https://doi.org/10.1145/1015864.1015884

[9] Muhammad Imran, Felix Kling, Stefano Soi, Florian Daniel, Fabio Casati, and Maurizio Marchese. 2012. ResEval Mash: A Mashup Tool for Advanced Research Evaluation. In Proceedings of the 21st International Conference on World Wide Web (WWW'12 Companion). ACM, New York, NY, USA, 361-364. https://doi.org/10. $1145 / 2187980.2188049$

[10] Andrew J. Ko, Robin Abraham, Laura Beckwith, Alan Blackwell, Margaret Burnett, Martin Erwig, Chris Scaffidi, Joseph Lawrance, Henry Lieberman, Brad Myers, Mary Beth Rosson, Gregg Rothermel, Mary Shaw, and Susan Wiedenbeck. 2011.
The State of the Art in End-user Software Engineering. ACM Comput. Surv. 43, 3 , Article 21 (April 2011), 44 pages. https://doi.org/10.1145/1922649.1922658

[11] Henry Lieberman, Fabio Paternò, Markus Klann, and Volker Wulf. 2006. End-User Development: An Emerging Paradigm. Springer Netherlands, Dordrecht, 1-8. https://doi.org/10.1007/1-4020-5386-X_1

[12] Catherine C. Marshall and Frank M. Shipman, III. 1995. Spatial Hypertext: Designing for Change. Commun. ACM 38, 8 (Aug. 1995), 88-97. https://doi.org/10. $1145 / 208344.208350$

[13] Anders I. Mørch, Gunnar Stevens, Markus Won, Markus Klann, Yvonne Dittrich, and Volker Wulf. 2004. Component-based Technologies for End-user Development. Commun. ACM 47, 9 (Sept. 2004), 59-62. https://doi.org/10.1145/1015864. 1015890

[14] Brad Myers, Scott E. Hudson, and Randy Pausch. 2000. Past, Present, and Future of User Interface Software Tools. ACM Trans. Comput.-Hum. Interact. 7, 1 (March 2000), 3-28. https://doi.org/10.1145/344949.344959

[15] Bonnie A. Nardi. 1993. A Small Matter of Programming: Perspectives on End User Computing (1st ed.). MIT Press, Cambridge, MA, USA.

[16] Fabio Paternò. 2013. End user development: Survey of an emerging field for empowering people. ISRN Software Engineering 2013 (2013). https://doi.org/10. $1155 / 2013 / 532659$

[17] Jochen Rode, Mary Beth Rosson, and Manuel A. Pérez QuiËIJnones. 2006. End User Development of Web Applications. Springer Netherlands, Dordrecht, 161-182. https://doi.org/10.1007/1-4020-5386-X_8

[18] C. Scaffidi, M. Shaw, and B. Myers. 2005. Estimating the Numbers of End Users and End User Programmers. In IEEE Symposium on Visual Languages and HumanCentric Computing (VL/HCC'05). IEEE. https://doi.org/10.1109/vlhcc.2005.34

[19] Thomas Schedel. 2016. Spatio-Temporal Parsing in Spatial Hypermedia. Ph.D. Dissertation. Aalborg University. https://opus4.kobv.de/opus4-hof/frontdoor/ index/index/docId/71

[20] Thomas Schedel and Claus Atzenbeck. 2016. Spatio-Temporal Parsing in Spatial Hypermedia. In Proceedings of the 27th ACM Conference on Hypertext and Social Media. ACM, 149-157. http://dx.doi.org/10.1145/2914586.2914596

[21] Yasuhiro Yamamoto, Kumiyo Nakakoji, and Atsushi Aoki. 2002. Spatial Hypertext for Linear-information Authoring: Interaction Design and System Development Based on the ART Design Principle. In Proceedings of the Thirteenth ACM Conference on Hypertext and Hypermedia (HYPERTEXT' 02 ). ACM, New York, NY, USA, 35-44. https://doi.org/10.1145/513338.513351

[22] Da-Qian Zhang and Kang Zhang. 1998. VisPro: a visual language generation toolset. In Proceedings. 1998 IEEE Symposium on Visual Languages (Cat. No.98TB100254). 195-202. https://doi.org/10.1109/VL.1998.706163 